\title{
Autosomal Recessive Hypercholesterolemia: A Rare Cause of Familial Hypercholesterolemia
}

\author{
Constantine E Kosmas ${ }^{1 *}$, Ian Martinez ${ }^{2}$, Rosmery Morcelo² ${ }^{2}$, Wellington Fabian ${ }^{3}$, Peter D Montan ${ }^{2}$, Eliscer \\ Guzman $^{4}$
}

${ }^{1}$ Department of Medicine, Division of Cardiology, Mount Sinai Hospital, New York, NY, USA

${ }^{2}$ Cardiology Clinic, Cardiology Unlimited, PC, New York, NY, USA

${ }^{3}$ Medical Clinic, Michael Correa, MD, PC, New York, NY, USA

${ }^{4}$ Department of Medicine, Division of Cardiology, Montefiore Medical Center, Bronx, NY, USA

Received: October 22, 2017; Published: October 26, 2017

*Corresponding author: Constantine E Kosmas, MD, PhD, 168-24 Powells Cove Blvd, Beechhurst, NY 11357, USA; Email: cekosmas1@gmail.com

\begin{abstract}
Autosomal recessive hypercholesterolemia (ARH) is a very rare genetic cause of hypercholesterolemia. ARH has been linked to mutations in the low-density lipoprotein receptor adaptor protein 1(LDLRAP1) with consequent disruption of the LDL receptor mediated endocytosis, leading to severe hypercholesterolemia. The clinical phenotype of ARH is milder than that of receptor-negative homozygous familial hypercholesterolemia (HoFH) and resembles that of receptor-defective HoFH. However, There is a large phenotypic variability in ARH and some ARH patients have LDL-cholesterol levels not significantly different from those of patients with HoFH. The prevalence of coronary artery disease, although increased, tends be lower in ARH compared to HoFH and patients with ARH, as compared to those with HoFH, tend to respond better to lipid-lowering drugs. This review aims to summarize the mechanism, as well as the genetic and clinical characteristics of ARH.
\end{abstract}

Keywords: Autosomal Recessive Hypercholesterolemia; Familial Hypercholesterolemia; LDL-Cholesterol; LDL Receptor

Abbreviations : ARH: Autosomal Recessive Hypercholesterolemia; LDLRAP1: Low-Density Lipoprotein Receptor Adaptor Protein 1; HoFH: Homozygous Familial Hypercholesterolemia; LDL-C: Low-Density Lipoprotein Cholesterol; CVD: Cardiovascular Disease; ApoB: Apolipoprotein B; PCSK9: Proprotein Convertase Subtilisin/Kexin Type 9; FH: Familial Hypercholesterolemia; CAD: Coronary Artery Disease; VLDL: Very Low Density Lipoprotein

\section{Introduction}

There are several genetic causes of hypercholesterolemia, which may lead to extremely high levels of total and low-density lipoprotein cholesterol (LDL-C) and subsequently to a significantly increased risk of cardiovascular disease (CVD). Mutations in the LDL receptor (LDLR) gene, the apolipoprotein $B(A p o B)$ gene and the proprotein convertase subtilisin/kexin type 9 (PCSK9) gene cause autosomal dominant hypercholesterolemia, which affects approximately in 1:250 to 1:500 individuals. On the other hand, autosomal recessive hypercholesterolemia (ARH) occurs much less frequently, estimated to occur in 1:1,000,000 live births, and has been linked to mutations in the gene that encodes the LDLR adaptor protein 1(LDLRAP1) [1].

This gene is found in the chromosomal region 1p36-p35 with a weight of 25 kilobases and it is formed by 9 exons which encode a 308-amino acid protein. In the autosomal recessive hypercholesterolemia, the endocytosis of the LDL receptor with its LDL ligand in the plasma membrane of the hepatocyte doesn't occur and the receptor-ligand complexes accumulate in the plasma membrane. As it was mentioned above, ARH is extremely rare with no more than 10,000 cases alive up to date, which are mostly found in the Lebanese, Mexican, Japanese, Indian, Turkish, Syrian and Italian populations [2]. Only 36 families with 14 different mutations have been reported in the literature until recently [3]. However, in some regions the prevalence of ARH appears to be higher and in the island of Sardinia the frequency of a heterozygous carrier mutation status for the LDLRAP1 gene is estimated to be approximately 1:143 individuals [3].

\section{Genetics and Mechanism}

The LDL receptor is the primary metabolic pathway for removal of cholesterol from the circulation. The ligand binding arm of the LDL-receptor binds to the ApoB protein present on the surface of LDL-cholesterol. This complex undergoes endocytosis in clathrincoated pits. PCSK9 is a circulating protein that is highly expressed in liver, small intestine, and kidney, and interacts with the extracellular 
domain of the LDL-receptor, targeting the receptor for degradation in the lysosome. More specifically, PCSK9 migrates to clathrincoated pits along with an LDL particle bound to an LDL receptor. The LDL-receptor-PCSK9 complex is internalized by vesicles that become endosomes with the aid of the LDLRAP1 (ARH protein) and delivers cholesterol to the cell. PCSK9 then accompanies the LDL receptor to the lysosome for catabolism [4].

Autosomal recessive hypercholesterolemia (ARH) is caused by recessive loss of function mutations in the LDLRAP1 gene, encoding LDLRAP1. Thus, internalization of the LDL-receptor via clathrincoated pits is aborted [5] and, as it was mentioned above, the complexes of the LDL receptor with its LDL ligand accumulate in the plasma membrane. Another endocytic clathrin adaptor protein, Disabled-2 (Dab2), may also act as an LDLR-selective adaptor. The activity and ability of Dab2 to mediate LDLR endocytosis have been demonstrated in cultured cells and this may be another site of mutation which can contribute to ARH [6].

\section{Clinical Phenotype of Patients with ARH}

Although the serum cholesterol levels of patients with ARH have been described as intermediate between familial hypercholesterolemia (FH) heterozygotes and $\mathrm{FH}$ homozygotes [7], there is clearly a large phenotypic variability in ARH. Some patients have 3-fold higher serum cholesterol levels than others, and their levels are not significantly different from those of classic FH homozygotes [7]. In some patients with ARH, large tuberous, tendinous or planar xanthomas are present, which are occasionally accompanied by corneal arcus and xanthelasmas [3,7].

In a study, which compared the clinical phenotype of 42 homozygous $\mathrm{FH}(\mathrm{HoFH})$ patients and $42 \mathrm{ARH}$ patients, it was found that the clinical phenotype of ARH is milder than that of receptornegative $\mathrm{HoFH}$ and resembles that of receptor-defective $\mathrm{HoFH}$. More specifically, the plasma LDL-C level was lower in ARH than in receptor-negative $\mathrm{HoFH}(14.25 \pm 2.29 \mathrm{mmol} / \mathrm{L}$ vs. $21.38 \pm 3.56$ $\mathrm{mmol} / \mathrm{L}$, respectively) but similar to that found in receptordefective HoFH $(15.52 \pm 2.39 \mathrm{mmol} / \mathrm{L})$. The risk of coronary artery disease (CAD) was 9-fold lower in ARH patients. No ARH patients $\leq$ 20 years of age were found to have CAD as opposed to $43 \%$ of HoFH. Furthermore, the prevalence of CAD was or tended to be lower in ARH in the $21-40$ (45\% versus $86 \%$ ) and $41-60$ (78\% versus $100 \%)$ age groups. Of note, heterozygous ARH carriers showed higher level of LDL-C (+17\%) than non-carrier family members [8].

\section{Lipid-lowering therapy in patients with ARH}

In general, $\mathrm{ARH}$ patients, as compared to those with $\mathrm{HoFH}$, tend to respond better to the lipid-lowering drugs. This may be due to the fact that the LDLR-dependent uptake of very low-density lipoprotein (VLDL) is maintained in the absence of LDLRAP1, which could likely contribute to the attenuation of the clinical phenotype of ARH [9]. Most of the times, a significant reduction of LDL-C levels occurs in patients with ARH after treatment with high-intensity statins alone or, more frequently, in combination with ezetimibe or bile acid sequestrants. Partial ileal bypass and apheresis at weekly or biweekly intervals have been also used with success in patients with ARH who do not reach target LDL-C concentrations on maximally tolerated dose of lipid-lowering drugs $[7,10]$. Since there is disruption of LDLR-mediated endocytosis, patients with ARH do not in general respond to PCSK9-inhibition therapy. However, a case of ARH responsive to a PCSK9 inhibitor (evolocumab) has been recently reported in the literature, which also suggests that the molecular implications of homozygous mutations in LDLRAP1 are distinct from homozygous receptor-negative LDL-C receptor states [11].

\section{References}

1. Stitziel NO, Fouchier SW, Sjouke B, Gina M Peloso, Alessa M Moscoso, et al. (2013) Exome sequencing and directed clinical phenotyping diagnose cholesterol ester storage disease presenting as autosomal recessive hypercholesterolemia. Arterioscler Thromb Vasc Biol 33(12): 2909-2914.

2. Matías Pérez D, Pérez Campos E, García-Montalvo IA (2015) A GENETIC VIEW OF FAMILIAL HYPERCHOLESTEROLEMIA. Nutr Hosp 32(6): 2421-2426.

3. Tada H, Kawashiri MA, Nohara A, Inazu A, Kobayashi J, et al. (2015) Autosomal recessive hypercholesterolemia: a mild phenotype of familial hypercholesterolemia: insight from the kinetic study using stable isotope and animal studies. J Atheroscler Thromb 22(1): 1-9.

4. Qian YW, Schmidt RJ, Zhang Y, Chu S, Lin A, et al. (2007) Secreted PCSK9 down regulates low density lipoprotein receptor through receptormediated endocytosis. J Lipid Res 48(7): 1488-1498.

5. De Castro Oros I, Pocovi M, Civeira F (2010) The genetic basis of familial hypercholesterolemia: inheritance, linkage, and mutations. Appl Clin Genet 3: 53-64.

6. Tao W, Moore R, Meng Y, Smith ER, Xu XX (2016) Endocytic adaptors Arh and Dab2 control homeostasis of circulatory cholesterol. J Lipid Res 57(5): 809-817.

7. Soutar AK, Naoumova RP, Traub LM (2003) Genetics, clinical phenotype, and molecular cell biology of autosomal recessive hypercholesterolemia. Arterioscler Thromb Vasc Biol 23(11): 1963-1970.

8. Pisciotta L, Priore Oliva C, Pes GM, Di Scala L, Bellocchio A, et al. (2006) Autosomal recessive hypercholesterolemia (ARH) and homozygous familial hypercholesterolemia (FH): a phenotypic comparison. Atherosclerosis 188(2): 398-405.

9. Jones C, Garuti R, Michaely P, Herz J, Hobbs HH, et al. (2007) Disruption of LDL but not VLDL clearance in autosomal recessive hypercholesterolemia. J Clin Invest 117(1): 165-174.

10. Muntoni S, Pisciotta L, Muntoni S, Bertolini S (2015) Pharmacological treatment of a Sardinian patient affected by Autosomal Recessive Hypercholesterolemia (ARH). J Clin Lipidol 9(1): 103-106.

11. Fahy EF, McCarthy E, Steinhagen Thiessen E, Vaughan CJ (2017) A case of autosomal recessive hypercholesterolemia responsive to proprotein convertase subtilisin/kexin 9 inhibition. J Clin Lipidol 11(1): 287-288. 


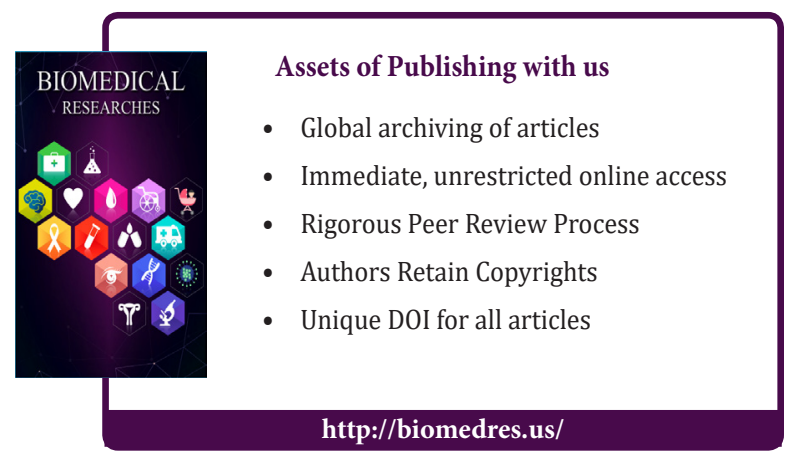

